\title{
Recursive Utility Maximization for Terminal Wealth under Partial Information
}

\author{
Shaolin Ji ${ }^{1,2}$ and Xiaomin Shi ${ }^{1}$ \\ ${ }^{1}$ Zhongtai Securities Institute for Financial Studies, Shandong University, Jinan 250100, China \\ ${ }^{2}$ Institute of Mathematics, Shandong University, Jinan 250100, China \\ Correspondence should be addressed to Xiaomin Shi; shixm@mail.sdu.edu.cn
}

Received 26 October 2016; Accepted 16 November 2016

Academic Editor: Weihai Zhang

Copyright (C) 2016 S. Ji and X. Shi. This is an open access article distributed under the Creative Commons Attribution License, which permits unrestricted use, distribution, and reproduction in any medium, provided the original work is properly cited.

This paper concerns the recursive utility maximization problem for terminal wealth under partial information. We first transform our problem under partial information into the one under full information. When the generator of the recursive utility is concave, we adopt the variational formulation of the recursive utility which leads to a stochastic game problem and characterization of the saddle point of the game is obtained. Then, we study the $K$-ignorance case and explicit saddle points of several examples are obtained. At last, when the generator of the recursive utility is smooth, we employ the terminal perturbation method to characterize the optimal terminal wealth.

\section{Introduction}

In this paper, we study the problem of an agent who invests in a financial market so as to maximize the recursive utility of his terminal wealth $X(T)$ on finite time interval $[0, T]$, while the recursive utility is characterized by the initial value $Y(0)$ of the following Backward stochastic differential equation (BSDE for short)

$$
\begin{aligned}
Y(t)= & u(X(T))+\int_{t}^{T} f(s, Y(s), Z(s)) d s \\
& -\int_{t}^{T} Z(s) d \widehat{W}(s) .
\end{aligned}
$$

The market consists of a riskless asset and $d$ risky assets, the latter being driven by a $d$-dimensional Brownian motion. And the investor has access only to the history of interest rates and prices of risky assets, while the appreciation rate and the driving Brownian motion are not directly observed. That is, the filtration generated by the Brownian motion could not be used when the investor chooses his portfolios. This is quite practical in a real financial market. So we are interested in this so-called recursive utility maximization problem under partial information.
In the full information case, the problem of maximizing the expected utility of terminal wealth is well understood in a complete or constrained financial market $[1,2]$. In an incomplete multiple-priors model, Quenez [3] studied the problem of maximization of utility of terminal wealth in which the asset prices are semimartingales. Schied [4] studied the robust utility maximization problem in a complete market under the existence of a "least favorable measure." As for the recursive utility optimization, El Karoui et al. [5] studied the optimization of recursive utilities when the generator of BSDE is smooth. Epstein and Ji $[6,7]$ formulated a model of recursive utility that captures the decision-maker's concern with ambiguity about both the drift and ambiguity and studied the recursive utility optimization under $G$-framework. $\mathrm{Hu}$ et al. [8] introduced a BSDE driven by G-Brownian motion from which a kind of more general recursive utility can be defined. Then $\mathrm{Hu}$ and Ji $[9,10]$ studied the corresponding control problem by two methods: maximum principle and dynamic programming principle. But all the above works do not accommodate partial information.

In the partial information case, Lakner [11] generalized the martingale method to expected utility maximization problem; see also Pham [12]. Cvitanić et al. [13] maximized 
the recursive utility under partial information. But the generator $f$ in Cvitanić et al. [13] does not depend on $z$. Miao [14] studied a special case of recursive multiple-priors utility maximization problem under partial information in which the appreciation rate is assumed to be an $\mathscr{F}_{0}$-measurable, unobserved random variable with known distribution. Actually, they studied the problem under Bayesian framework and did not give the explicit solutions.

In this paper, we first transform our portfolio selection problem under partial information into one under full information in which the unknown appreciation rate is replaced by its filter estimate and the Brownian motion is replaced by the innovation process. Then a backward formulation of the problem under full information is built in which instead of the portfolio process, the terminal wealth is regarded as the control variable. This backward formulation is based on the existence and uniqueness theorem of BSDE and was introduced in $[5,15]$.

When the generator $f$ of (1) is concave, we adopt the variational formulation of the recursive utility which leads to a stochastic game problem. Inspired by the convexity duality method developed in Cvitanić and Karatzas [16], we turn the primal "sup-inf" problem to a dual minimization problem over a set of discounting factors and equivalent probability measures. A characterization of the saddle point of this game is obtained in this paper. Furthermore, the explicit saddle points for several classical examples are worked out.

When the generator $f$ of the BSDE is smooth, we apply the terminal perturbation method developed in Ji and Zhou [17] and Ji and Peng [18] to characterize the optimal terminal wealth of the investor. Once the optimal terminal wealth is obtained, the determination of the optimal portfolio process is a martingale representation problem which we do not involve in this paper.

The rest of this paper is organized as follows. In Section 2, we formulate the recursive utility maximization problem under partial information, reduce the original problem to a problem under full information, and give the backward formulation. The case of nonsmooth generator is tackled in Section 3. In Section 4, we specialize in $K$-ignorance model and give explicit saddle points of several examples. In Section 5, we characterize the optimal wealth when the generator $f$ is smooth.

\section{The Problem of Recursive Utility Maximization under Partial Observation}

2.1. Classical Formulation of the Problem. We consider a financial market consisting of a riskless asset whose price process is assumed for simplicity to be equal to one and $d$ risky securities (the stocks) whose prices are stochastic processes $S_{i}(t), i=0,1, \ldots, d$ governed by the following SDEs:

$$
\begin{aligned}
& d S_{i}(t)=S_{i}(t)\left(\mu_{i}(t) d t+\sum_{j=1}^{d} \sigma_{i j}(t) d W_{j}(t)\right) \\
& i=1, \ldots, d,
\end{aligned}
$$

where $W(\cdot)=\left(W_{1}(\cdot), \ldots, W_{d}(\cdot)\right)^{\prime}$ is a standard $d$-dimensional Brownian motion defined on a filtered complete probability $\operatorname{space}\left(\Omega, \mathscr{F},\left\{\mathscr{F}_{t}\right\}_{t \geq 0}, P\right) . \mu^{\prime}=\left\{\mu^{\prime}(t)=\left(\mu_{1}(t), \ldots, \mu_{d}(t)\right), t \in\right.$ $[0, T]\}$ is the appreciation rate of the stocks which is $\mathscr{F}_{t^{-}}$ adapted, bounded, and the $d \times d$ matrix $\sigma(t)=\left(\sigma_{i j}(t)\right)_{1 \leq i, j \leq d}$ is the disperse rate of the stocks. Here and throughout the paper ' denotes the transpose operator.

The asset prices are assumed to be continuously observed by the investors in this market; in other words, the information available to the investors is represented by $\mathbb{G}=\left\{\mathscr{G}_{t}\right\}_{t>0}$, which is the $P$-augmentation of the filtration generated by the price processes $\sigma(S(u) ; 0 \leq u \leq t)$. The matrix disperse coefficient $\sigma(t)$ is assumed invertible, bounded uniformly, and $\exists \varepsilon>0, \rho^{\prime} \sigma(t) \sigma^{\prime}(t) \rho \geq \varepsilon\|\rho\|^{2}, \forall \rho \in \mathbb{R}^{d}, t \in[0, T]$, a.s. In fact, $\sigma(t)$ can be obtained from the quadratic variation of the price process. So we assume w.l.o.g. that $\sigma(t)$ is $\mathscr{G}_{t}$-adapted. However, the appreciation rate $\mu^{\prime}(t):=\left(\mu_{1}(t), \ldots, \mu_{d}(t)\right)$ is not observable for the investors.

A small investor whose actions cannot affect the market prices can decide at time $t \in[0, T]$ what amount $\pi_{i}(t)$ of his wealth to invest in the $i$ th stock, $i=1, \ldots, d$. Of course, his decision can only be based on the available information $\left\{\mathscr{G}_{t}\right\}_{t=0}^{T}$; that is, the processes $\pi^{\prime}(\cdot)=\left(\pi_{1}(\cdot), \ldots, \pi_{d}(\cdot)\right)$ : $[0, T] \times \Omega \rightarrow \mathbb{R}^{d}$ are $\left\{\mathscr{G}_{t}\right\}_{t=0}^{T}$ progressively measurable and satisfy $E \int_{0}^{T}\|\pi(t)\|^{2} d t<\infty$.

Then the wealth process $X(\cdot) \equiv X^{x, \pi}(\cdot)$ of a self-financing investor who is endowed with initial wealth $x>0$ satisfies the following stochastic differential equation:

$$
\begin{aligned}
d X(t) & =\sum_{i=1}^{d} \pi_{i}(t) \frac{d S_{i}(t)}{S_{i}(t)} \\
& =\pi^{\prime}(t) \mu(t) d t+\pi^{\prime}(t) \sigma(t) d W(t) .
\end{aligned}
$$

Because the only information available to the investor is $\mathbb{G}$, we could not use the Brownian motion $W$ to define the recursive utility. As we will show in the following, there exists a Brownian motion $\widehat{W}$ under $P$ in the filtered measurable space $(\Omega, \mathbb{G})$ which is often referred to as an innovation process. The recursive utility process $Y(t) \equiv Y^{x, \pi}(t)$ of the investor is defined by the following backward stochastic differential equation:

$$
\begin{aligned}
Y(t)= & u(X(T))+\int_{t}^{T} f(s, Y(s), Z(s)) d s \\
& -\int_{t}^{T} Z(s) d \widehat{W}(s),
\end{aligned}
$$

where $f$ and $u$ are functions satisfying the following assumptions.

Assumption 1. (A1) $f: \Omega \times[0, T] \times \mathbb{R} \times \mathbb{R}^{d} \rightarrow \mathbb{R}$ is $\mathbb{G}$ progressively measurable for any $(y, z) \in \mathbb{R} \times \mathbb{R}^{d}$.

(A2) There exists a constant $C \geq 0$ such that

$$
\begin{aligned}
& \left|f\left(t, y_{1}, z_{1}\right)-f\left(t, y_{2}, z_{2}\right)\right| \leq C\left(\left|y_{1}-y_{2}\right|+\left|z_{1}-z_{2}\right|\right), \\
& \forall\left(t, \omega, y_{1}, y_{2}, z_{1}, z_{2}\right) \in \Omega \times[0, T] \times \mathbb{R} \times \mathbb{R} \times \mathbb{R}^{d} \times \mathbb{R}^{d} .
\end{aligned}
$$


(A3) $f(t, \cdot, \cdot)$ is continuous about $t$ and $E \int_{0}^{T} f^{2}(t, 0,0) d t<$ $+\infty$.

Assumption 2. $u: \mathbb{R}^{+} \rightarrow \mathbb{R}$ is continuously differentiable and satisfies linear growth condition.

Remark 3. Equation (4) is not a standard BSDE because in general $\mathbb{G}$ is strictly larger than the augmented filtration of the $(P, \mathbb{G})$-Brownian motion $\widehat{W}$.

We introduce the following spaces:

$L^{2}\left(\Omega, \mathscr{G}_{T}, P ; \mathbb{R}\right):=\left\{\xi: \Omega \longrightarrow \mathbb{R} \mid \xi\right.$ is $\mathscr{G}_{T}$-measurable,

$\left.E|\xi|^{2}<\infty\right\}$

$M_{\mathbb{G}}^{2}\left(0, T ; \mathbb{R}^{d}\right):=\left\{\phi:[0, T] \times \Omega \longrightarrow \mathbb{R}^{d} \mid\left(\phi_{t}\right)_{0 \leq t \leq T}\right.$

is $\mathbb{G}$-progressively measurable process, $\|\phi\|^{2}$

$\left.=E \int_{0}^{T}|\phi(t)|^{2} d t<\infty\right\}$,

$S_{\mathbb{G}}^{2}(0, T ; \mathbb{R}):=\left\{\phi:[0, T] \times \Omega \longrightarrow \mathbb{R} \mid\left(\phi_{t}\right)_{0 \leq t \leq T}\right.$

is $\mathbb{G}$-progressively measurable process, $\|\phi\|_{S}^{2}$

$$
\left.=E\left[\sup _{0 \leq t \leq T}|\phi(t)|^{2}\right]<\infty\right\} \text {. }
$$

For notational simplicity, we will often write $L_{\mathscr{G}_{T}}^{2}, M_{\mathbb{G}}^{2}$ and $S_{\mathbb{G}}^{2}$ instead of $L^{2}\left(\Omega, \mathscr{G}_{T}, P ; \mathbb{R}\right), M_{\mathbb{G}}^{2}\left(0, T ; \mathbb{R}^{d}\right)$, and $S_{\mathbb{G}}^{2}(0, T ; \mathbb{R})$, respectively.

We will show in the next subsection that under Assumption 1 , for any $\xi \in L_{\mathscr{G}_{T}}^{2}$, the BSDE (4) has a unique solution $(Y(\cdot), Z(\cdot)) \in S_{\mathbb{G}}^{2} \times M_{\mathbb{G}}^{2}$. Then for each $\pi \in M_{\mathbb{G}}^{2}, X(T) \in L_{\mathscr{G}_{\mathrm{T}}}^{2}$, and Assumption 2 ensures that the variable $u(X(T)) \in L_{\mathscr{G}_{T}}^{2}$. Thus, under Assumptions 1 and 2, the recursive utility process associated with this terminal value is well defined.

Given an utility function satisfying Assumption 2 and initial endowment $x$, the recursive utility maximization problem with bankruptcy prohibition is formulated as the investor chooses a portfolio strategy so as to

$$
\begin{aligned}
\text { Maximize } & Y^{x, \pi}(0), \\
\text { s.t. } \quad & X(t) \geq 0, \quad t \in[0, T] \text {, a.s., } \\
& \pi(\cdot) \in M_{\mathbb{G}}^{2}, \\
& (X(\cdot), \pi(\cdot)) \text { satisfies Eq. (3), } \\
& (Y(\cdot), Z(\cdot)) \text { satisfies Eq. (4), }
\end{aligned}
$$

where $X(t) \geq 0$ means that no-bankruptcy is required.

Definition 4. A portfolio $\pi(\cdot)$ is said to be admissible if $\pi(\cdot) \epsilon$ $M_{\mathbb{G}}^{2}$ and the corresponding wealth processes $X(t) \geq 0, t \in$ $[0, T]$, a.s.
Given initial wealth $x>0$, denote by $\overline{\mathscr{A}}(x)$ the set of investor's feasible portfolio strategies; that is

$$
\overline{\mathscr{A}}(x)=\left\{\pi: \pi \in M_{\mathbb{G}}^{2}, X^{x, \pi}(t) \geq 0, d P \otimes d t \text { a.s. }\right\} .
$$

2.2. Reduction to a Problem under Full Information. Define the risk premium process $\eta(t)=\sigma(t)^{-1} \mu(t)$. Because we have assumed the processes $\mu(\cdot)$ and $\sigma(\cdot)$ are uniformly bounded, the process

$$
L(t):=\exp \left(-\int_{0}^{t} \eta^{\prime}(s) d W(s)-\frac{1}{2} \int_{0}^{t}|\eta(s)|^{2} d s\right)
$$

is a $(P, \mathbb{F})$ martingale. So a probability measure $\widetilde{P}$ can be defined by

$$
\widetilde{P}(A)=E\left[L(T) I_{A}\right]
$$

$$
\forall A \in \mathscr{F}_{T} \text {, where } \frac{d \widetilde{P}}{d P}=L(T) .
$$

$\widetilde{P}$ is usually called risk neutral probability in the financial market. The process

$$
\widetilde{W}(t):=W(t)+\int_{0}^{t} \eta(s) d s, \quad 0 \leq t \leq T
$$

is a Brownian motion under $\widetilde{P}$ by Girsanov's theorem.

Then we can rewrite the stock price processes (2) as

$$
d S_{i}(t)=S_{i}(t)\left(\sum_{j=1}^{d} \sigma_{i j}(t) d \widetilde{W}_{j}(t)\right), \quad i=1, \ldots, d
$$

Note that $\sigma(t)$ is assumed to be bounded, invertible, and $\mathscr{G}_{t}$-adapted. So the filtration $\mathbb{G}$ coincides with the augmented natural filtration of $\widetilde{W}$ by Theorem V.3.7 in [19].

Let $\widehat{\eta}(t):=E\left[\eta(t) \mid \mathscr{G}_{t}\right]$ be a measurable version of the conditional expectation of $\eta$ with respect to the filtration $\mathbb{G}$. Set $\widehat{\mu}: \widehat{\mu}(t)=E\left[\mu(t) \mid \mathscr{G}_{t}\right]$. Then $\widehat{\mu}(t)=\sigma(t) \widehat{\eta}(t)$, since $\sigma$ is $\mathbb{G}$-adapted.

We introduce the process

$$
\begin{aligned}
\widehat{W}(t) & :=\widetilde{W}(t)-\int_{0}^{t} \widehat{\eta}(s) d s \\
& =W(t)+\int_{0}^{t}(\eta(s)-\widehat{\eta}(s)) d s, \quad t \geq 0 .
\end{aligned}
$$

By Theorem 8.1.3 and Remark 8.1.1 in Kallianpur [20], $\{\widehat{W}(t), t \geq 0\}$ is a $(\mathbb{G}, P)$-Brownian motion which is the so-called innovations process. Then, we could describe the dynamics of stock price processes and the wealth process within a full observation model:

$$
\begin{gathered}
d S_{i}(t)=S_{i}(t)\left(\widehat{\mu}_{i}(t) d t+\sum_{j=1}^{d} \sigma_{i j}(t) d \widehat{W}_{j}(t)\right), \\
\quad i=1, \ldots, d, \\
d X(t)=\pi^{\prime}(t) \hat{\mu}(t) d t+\pi^{\prime}(t) \sigma(t) d \widehat{W}(t) .
\end{gathered}
$$


Now all the coefficients in our model are observable. So we are in a full observation model and our problem (7) can be reformulated as follows:

$$
\begin{aligned}
\text { Maximize } & Y^{x_{0}, \pi}(0), \\
\text { s.t. } \quad & X(t) \geq 0 \quad \forall t \in[0, T] \text { a.s., } \\
& \pi(\cdot) \in M_{\mathbb{G}}^{2}, \\
& (X(\cdot), \pi(\cdot)) \text { satisfies Eq. (3), } \\
& (Y(\cdot), Z(\cdot)) \text { satisfies Eq. (4). }
\end{aligned}
$$

2.3. Backward Formulation of the Problem. In this subsection, we first show BSDE (4) has a unique solution under some mild conditions and then give an equivalent backward formulation of problem (15).

Lemma 5. Under Assumption 1, for $\forall \xi \in L_{\mathbb{G}}^{2}$, there exists a unique solution $(Y, Z) \in S_{\mathbb{G}}^{2} \times M_{\mathbb{G}}^{2}$ to the $\operatorname{BSDE}(4)$.

Since $\mathbb{G}$ is strictly larger than the augmented filtration of the $(P, \mathbb{G})$-Brownian motion $\widehat{W}$ in general, equation (4) is not a standard BSDE. Fortunately, by Theorem 8.3.1 in [20], every square integrable $\mathscr{G}_{t}$-martingale $M(t)$ can be represented as

$$
M(t)=M(0)+\int_{0}^{t} Z^{\prime}(s) d \widehat{W}(s),
$$

where $Z(\cdot) \in M_{\mathbb{G}}^{2}$. Thus, applying similar analysis as in [21], it is easy to prove this lemma.

Let $q(\cdot):=\sigma(\cdot)^{\prime} \pi(\cdot)$. Since $\sigma(\cdot)$ is invertible, $q(\cdot)$ can be regarded as the control variable instead of $\pi(\cdot)$. By the existence and uniqueness result of BSDE (4), selecting $q(\cdot)$ is equivalent to selecting the terminal wealth $X(T)$. If we take the terminal wealth as control variable, the wealth equation and recursive utility process can be written as

$$
\begin{aligned}
-d X(t) & =-q^{\prime-1}(t) \widehat{\mu}(t) d t-q^{\prime}(t) d \widehat{W}(t), \\
X(T) & =\xi, \\
-d Y(t) & =f(t, Y(t), Z(t)) d t-Z^{\prime}(t) d \widehat{W}(t), \\
Y(T) & =u(\xi),
\end{aligned}
$$

where the "control" is the terminal wealth $\xi$ to be chosen from the following set:

$$
U:=\left\{\xi \mid \xi \in L_{\mathscr{G}_{T}}^{2}, \xi \geq 0\right\}
$$

From now on, we denote the solution of (17) by $\left(X^{\xi}(\cdot), q^{\xi}(\cdot), Y^{\xi}(\cdot), Z^{\xi}(\cdot)\right)$. We also denote $X^{\xi}(0)$ and $Y^{\xi}(0)$ by $X_{0}^{\xi}$ and $Y_{0}^{\xi}$, respectively.

As implied by the comparison theorem for BSDE (4), the nonnegative terminal wealth $(\xi=X(T) \geq 0)$ keeps the wealth process nonnegative all the time. This gives rise to the following optimization problem:

$$
\begin{aligned}
\text { Maximize } & J(\xi):=Y_{0}^{\xi}, \\
\text { s.t. } \quad & \xi \in U, \\
& X_{0}^{\xi}=x, \\
& \left(X^{\xi}(\cdot), q^{\xi}(\cdot)\right),\left(Y^{\xi}(\cdot), Z^{\xi}(\cdot)\right) \text { satisfies Eq. }(17) .
\end{aligned}
$$

Definition 6. A random variable $\xi \in U$ is called feasible for the initial wealth $x$ if and only if $X^{\xi}(0)=x$. We will denote the set of all feasible $\xi$ for the initial wealth $x$ by $\mathscr{A}(x)$.

It is clear that original problems (7) and (15) are equivalent to the auxiliary one (19). Hence, hereafter we focus ourselves on solving (19). Note that $\xi$ becomes the control variable. The advantage of this approach is that the state constraint in (7) becomes a control constraint in (19), whereas it is well known in control theory that a control constraint is much easier to deal with than a state constraint. The cost of this approach is the original initial condition $X^{\xi}(0)=x$ that now becomes a constraint.

Feasible $\xi^{*} \in \mathscr{A}(x)$ is called optimal if it attains the maximum of $J(\xi)$ over $\mathscr{A}(x)$. Once $\xi^{*}$ is determined, the optimal portfolio can be obtained by solving the first equation in (17) with $X^{\xi^{*}}(T)=\xi^{*}$.

\section{Dual Method for Recursive Utility Maximization}

In this section, we impose the following concavity condition.

Assumption 7. The function $(y, z) \mapsto f(\omega, t, y, z)$ is concave for all $(\omega, t) \in \Omega \times[0, T]$.

We also need the following assumption on $u$.

Assumption 8. $u:(0, \infty) \rightarrow \mathbb{R}$ is strictly increasing, strictly concave, and continuously differentiable, and satisfies

$$
\begin{aligned}
& u^{\prime}(0+):=\lim _{x \downarrow 0} u^{\prime}(x)=\infty, \\
& u^{\prime}(\infty):=\lim _{x \rightarrow \infty} u^{\prime}(x)=0 .
\end{aligned}
$$

Under Assumption 8, Assumption 2 seems too restrictive and it precludes some interesting examples. So in the following 
two sections, for any given utility function $u$ satisfying Assumption 8, we set

$$
U=\left\{\xi \mid \xi \in L_{\mathscr{G}_{T}}^{2}, u(\xi) \in L_{\mathscr{G}_{T}}^{2}, \xi \geq 0\right\} .
$$

In this section, we assume $\sigma \equiv I_{d}$, the $d$-dimensional identity matrix. Let $F(t, \beta, \gamma)$ be the Fenchel-Legendre transform of $f$ :

$$
\begin{array}{r}
F(\omega, t, \beta, \gamma):=\sup _{(y, z) \in \mathbb{R} \times \mathbb{R}^{d}}\left[f(\omega, t, y, z)-y \beta-z^{\prime} \gamma\right], \\
(\beta, \gamma) \in \mathbb{R} \times \mathbb{R}^{d} .
\end{array}
$$

Let the effective domain of $F$ be $\mathscr{D}_{F}:=\{(\omega, t, \beta, \gamma) \in \Omega \times$ $\left.[0, T] \times \mathbb{R} \times \mathbb{R}^{d} \mid F(\omega, t, \beta, \gamma)<+\infty\right\}$. As was shown in [22], the $(\omega, t)$-section of $\mathscr{D}_{F}$, denoted by $\mathscr{D}_{F}^{(\omega, t)}$ is included in the bounded domain $B=[-C, C]^{d+1} \subset \mathbb{R} \times \mathbb{R}^{d}$, where $C$ is the Lipschitz constant of $f$.

We have the duality relation by the concavity of $f$,

$$
f(\omega, t, y, z)=\inf _{(\beta, \gamma) \in \mathscr{D}_{F}^{(\omega, t)}}\left[F(\omega, t, \beta, \gamma)+y \beta+z^{\prime} \gamma\right] .
$$

For every $(\omega, t, y, z)$ the infimum is achieved in this relation by a pair $(\beta, \gamma)$ which depends on $(\omega, t)$.

Set

$$
\mathscr{B}=\{(\beta, \gamma) \mid(\beta, \gamma) \text { is } \mathbb{G} \text {-progressively measurable, }
$$

$$
\left.B \text {-valued, } E \int_{0}^{T} F\left(t, \beta_{t}, \gamma_{t}\right)^{2} d t<+\infty\right\} \text {. }
$$

Then $\mathscr{B}$ is a convex set. For any $(\beta, \gamma) \in \mathscr{B}$, let

$$
f^{\beta, \gamma}(t, y, z)=F\left(t, \beta_{t}, \gamma_{t}\right)+y \beta_{t}+z^{\prime} \gamma_{t},
$$

and denote by $\left(Y^{\beta, \gamma}, Z^{\beta, \gamma}\right)$ the unique solution to the linear $\operatorname{BSDE}(4)$ with $f^{\beta, \gamma}$.

By similar analysis as Proposition 3.4 in [22], we have the following variational formulation of $X^{\xi}(t)$ and $Y^{\xi}(t)$.

Lemma 9. Under Assumptions 1 and 7 , for any $\xi \in U$, the solutions $\left(X^{\xi}(\cdot), q^{\xi}(\cdot)\right),\left(Y^{\xi}(\cdot), Z^{\xi}(\cdot)\right)$ of $(17)$ can be represented as

$$
\begin{aligned}
X^{\xi}(t) & =\widehat{L}^{-1}(t) E\left[\widehat{L}(T) \xi \mid \mathscr{G}_{t}\right], \\
Y^{\xi}(t) & =\text { ess } \inf _{(\beta, \gamma) \in \mathscr{B}} Y_{t}^{\beta, \gamma}, \quad t \in[0, T], \text { a.s. }
\end{aligned}
$$

where

$$
\begin{aligned}
\widehat{L}(t) & :=e^{-\int_{0}^{t} \widehat{\mu}^{\prime}(s) d \widehat{W}(s)-(1 / 2) \int_{0}^{t}|\widehat{\mu}(s)|^{2} d s}, \\
Y_{t}^{\beta, \gamma} & =E\left[\int_{t}^{T} \Gamma_{t, s}^{\beta, \gamma} F\left(s, \beta_{s}, \gamma_{s}\right) d s+\Gamma_{t, T}^{\beta, \gamma} u(\xi) \mid \mathscr{G}_{t}\right], \\
\Gamma_{t, s}^{\beta, \gamma} & =e^{\int_{t}^{s}\left(\beta_{r}-(1 / 2)\left|\gamma_{r}\right|^{2}\right) d r+\int_{t}^{s} \gamma_{r}^{\prime} d \widehat{W}(r)} .
\end{aligned}
$$

In particular, we have $Y^{\xi}(0)=\inf _{(\beta, \gamma) \in \mathscr{B}} E\left[\int_{0}^{T} \Gamma_{0, s}^{\beta, \gamma} F\left(s, \beta_{s}\right.\right.$, $\left.\left.\gamma_{s}\right) d s+\Gamma_{0, T}^{\beta, \gamma} u(\xi)\right]$
Remark 10. By Theorem 3.1 in [11], we have $\widehat{L}(t)=$ $E\left[L(t) \mid \mathscr{G}_{t}\right], t \in[0, T]$, a.s.

By Lemma 9, $\mathscr{A}(x)=\{\xi \in U \mid E[\widehat{L}(T) \xi]=x\}$. Thus, our problem is equivalent to the following problem:

Maximize $J(\xi)$

$$
\begin{aligned}
& =\inf _{(\beta, \gamma) \in \mathscr{B}} E\left[\int_{0}^{T} \Gamma_{0, s}^{\beta, \gamma} F\left(s, \beta_{s}, \gamma_{s}\right) d s+\Gamma_{0, T}^{\beta, \gamma} u(\xi)\right] \\
\text { s.t. } \quad \xi & \in \mathscr{A}(x) .
\end{aligned}
$$

The maximum recursive utility that the investor can achieve is

$$
\begin{aligned}
& \underline{V}(x):=\sup _{\xi \in \mathscr{A}(x)} \inf _{(\beta, \gamma) \in \mathscr{B}} E\left[\int_{0}^{T} \Gamma_{0, s}^{\beta, \gamma} F\left(s, \beta_{s}, \gamma_{s}\right) d s\right. \\
& \left.\quad+\Gamma_{0, T}^{\beta, \gamma} u(\xi)\right] .
\end{aligned}
$$

It is dominated by its "min-max" counterpart

$$
\begin{aligned}
& \bar{V}(x):=\inf _{(\beta, \gamma) \in \mathscr{B}} \sup _{\xi \in \mathscr{A}(x)} E\left[\int_{0}^{T} \Gamma_{0, s}^{\beta, \gamma} F\left(s, \beta_{s}, \gamma_{s}\right) d s\right. \\
& \left.\quad+\Gamma_{0, T}^{\beta, \gamma} u(\xi)\right] .
\end{aligned}
$$

If we can find $(\widehat{\beta}, \widehat{\gamma}, \widehat{\xi}) \in \mathscr{B} \times \mathscr{A}(x)$ such that

$$
\begin{aligned}
\underline{V}(x) & =E\left[\int_{0}^{T} \Gamma_{0, s}^{\widehat{\beta}, \widehat{\gamma}} F\left(s, \widehat{\beta}_{s}, \widehat{\gamma}_{s}\right) d s+\Gamma_{0, T}^{\widehat{\beta}, \widehat{\gamma}} u(\widehat{\xi})\right] \\
& =\bar{V}(x),
\end{aligned}
$$

then the optimal solution of problem (28) is $\widehat{\xi}$.

Let us introduce the monotone decreasing function $I(\cdot)$ as the inverse of the marginal utility function $u^{\prime}(\cdot)$ and the convex dual

$$
\tilde{u}(\zeta):=\max _{x>0}[u(x)-\zeta x]=u(I(\zeta))-\zeta I(\zeta), \quad \zeta>0 .
$$

Then, $\forall \xi \in \mathscr{A}(x), \forall(\beta, \gamma) \in \mathscr{B}, \forall \zeta>0$,

$$
\begin{aligned}
& E\left[\int_{0}^{T} \Gamma_{0, s}^{\beta, \gamma} F\left(s, \beta_{s}, \gamma_{s}\right) d s+\Gamma_{0, T}^{\beta, \gamma} u(\xi)\right] \\
& \quad \leq E\left[\int_{0}^{T} \Gamma_{0, s}^{\beta, \gamma} F\left(s, \beta_{s}, \gamma_{s}\right) d s+\Gamma_{0, T}^{\beta, \gamma} \widetilde{u}\left(\zeta \frac{\widehat{L}(T)}{\Gamma_{0, T}^{\beta, \gamma}}\right)\right. \\
& +\zeta \xi \widehat{L}(T)]=E\left[\int_{0}^{T} \Gamma_{0, s}^{\beta, \gamma} F\left(s, \beta_{s}, \gamma_{s}\right) d s\right. \\
& \left.\quad+\Gamma_{0, T}^{\beta, \gamma} \widetilde{u}\left(\zeta \frac{\widehat{L}(T)}{\Gamma_{0, T}^{\beta, \gamma}}\right)\right]+\zeta x .
\end{aligned}
$$


Furthermore, we have equality in the above formula for some $\widehat{\xi} \in \mathscr{A}(x),(\widehat{\beta}, \widehat{\gamma}) \in \mathscr{B}, \widehat{\zeta}>0$ if and only if the conditions

$$
\begin{aligned}
E[\widehat{\xi} \widehat{L}(T)] & =x, \\
\widehat{\xi} & =I\left(\widehat{\zeta} \frac{\widehat{L}(T)}{\Gamma_{0, T}^{\widehat{\beta}, \widehat{\gamma}}}\right), \text { a.s. }
\end{aligned}
$$

are satisfied simultaneously. And in this case, we have

$$
\begin{aligned}
& E\left[\int_{0}^{T} \Gamma_{0, s}^{\widehat{\beta}, \widehat{\gamma}} F\left(s, \widehat{\beta}_{s}, \widehat{\gamma}_{s}\right) d s+\Gamma_{0, T}^{\hat{\beta}, \widehat{\gamma}^{2}} u(\widehat{\xi})\right] \\
& =E\left[\int_{0}^{T} \Gamma_{0, s}^{\hat{\beta}, \widehat{\gamma}} F\left(s, \widehat{\beta}_{s}, \widehat{\gamma}_{s}\right) d s+\Gamma_{0, T}^{\hat{\beta}, \widehat{u}} \widetilde{u}\left(\widehat{\zeta} \frac{\widehat{L}(T)}{\Gamma_{0, T}^{\widehat{\beta}, \widehat{\gamma}}}\right)\right] \\
& +\widehat{\zeta} x \text {. }
\end{aligned}
$$

Lemma 11. Under Assumptions 1, 7, anfd 8, suppose that there exists a quadruple $(\widehat{\xi}, \widehat{\beta}, \widehat{\gamma}, \widehat{\zeta}) \in(\mathscr{A}(x) \times \mathscr{B} \times(0, \infty))$ which satisfies (34) and

$$
\begin{aligned}
& E\left[\int_{0}^{T} \Gamma_{0, s}^{\widehat{\beta}, \widehat{\gamma}} F\left(s, \widehat{\beta}_{s}, \widehat{\gamma}_{s}\right) d s+\Gamma_{0, T}^{\hat{\beta}, \widehat{\gamma}} u(\widehat{\xi})\right] \\
& \leq E\left[\int_{0}^{T} \Gamma_{0, s}^{\beta, \gamma} F\left(s, \beta_{s}, \gamma_{s}\right) d s+\Gamma_{0, T}^{\beta, \gamma} u(\widehat{\xi})\right], \\
& \forall(\beta, \gamma) \in \mathscr{B} .
\end{aligned}
$$

Then we have $\forall \xi \in \mathscr{A}(x), \forall(\beta, \gamma) \in \mathscr{B}$, and

$$
\begin{aligned}
& E\left[\int_{0}^{T} \Gamma_{0, s}^{\widehat{\beta}, \widehat{\gamma}} F\left(s, \widehat{\beta}_{s}, \widehat{\gamma}_{s}\right) d s+\Gamma_{0, T}^{\hat{\beta}, \widehat{\gamma}} u(\xi)\right] \\
& \quad \leq E\left[\int_{0}^{T} \Gamma_{0, s}^{\hat{\beta}, \widehat{\gamma}} F\left(s, \widehat{\beta}_{s}, \widehat{\gamma}_{s}\right) d s+\Gamma_{0, T}^{\hat{\beta}, \widehat{\gamma}} u(\widehat{\xi})\right] \\
& \quad \leq E\left[\int_{0}^{T} \Gamma_{0, s}^{\beta, \gamma} F\left(s, \beta_{s}, \gamma_{s}\right) d s+\Gamma_{0, T}^{\beta, \gamma} u(\widehat{\xi})\right] .
\end{aligned}
$$

That is, $(\widehat{\xi}, \widehat{\beta}, \widehat{\gamma})$ is a saddle point satisfying (31).

Proof. We only prove the first relationship in (37). Let $(\beta, \gamma)=$ $(\widehat{\beta}, \widehat{\gamma})$ and $\zeta=\widehat{\zeta}$ in $(33)$. We get

$$
\begin{aligned}
& E\left[\int_{0}^{T} \Gamma_{0, s}^{\hat{\beta}, \widehat{\gamma}} F\left(s, \widehat{\beta}_{s}, \widehat{\gamma}_{s}\right) d s+\Gamma_{0, T}^{\widehat{\beta}, \widehat{\gamma}} u(\xi)\right] \\
& \leq E\left[\int_{0}^{T} \Gamma_{0, s}^{\hat{\beta}, \widehat{\gamma}} F\left(s, \widehat{\beta}_{s}, \widehat{\gamma}_{s}\right) d s+\Gamma_{0, T}^{\widehat{\beta}, \widehat{\psi}} \widetilde{u}\left(\widehat{\zeta} \frac{\widehat{L}(T)}{\Gamma_{0, T}^{\widehat{\beta}, \widehat{\gamma}}}\right)\right] \\
& +\widehat{\zeta} x=E\left[\int_{0}^{T} \Gamma_{0, s}^{\hat{\beta}, \hat{\gamma}} F\left(s, \widehat{\beta}_{s}, \widehat{\gamma}_{s}\right) d s+\Gamma_{0, T}^{\widehat{\beta}, \widehat{\gamma}} u(\widehat{\xi})\right],
\end{aligned}
$$

$\forall \xi \in \mathscr{A}(x)$,
Let us introduce the value function

$$
\begin{aligned}
& \widetilde{V}(\zeta) \equiv \widetilde{V}(\zeta ; x):=\inf _{(\beta, \gamma) \in \mathscr{B}} E\left[\int_{0}^{T} \Gamma_{0, s}^{\beta, \gamma} F\left(s, \beta_{s}, \gamma_{s}\right) d s\right. \\
& \left.+\Gamma_{0, T}^{\beta, \gamma} \widetilde{\mathcal{u}}\left(\zeta \frac{\widehat{L}(T)}{\Gamma_{0, T}^{\beta, \gamma}}\right)\right], \quad 0<\zeta<\infty .
\end{aligned}
$$

By (33), we have

$$
\bar{V}(x) \leq V_{*}(x),
$$

where

$$
\begin{aligned}
& V_{*}(x):=\inf _{\zeta>0,(\beta, \gamma) \in \mathscr{B}} E\left[\int_{0}^{T} \Gamma_{0, s}^{\beta, \gamma} F\left(s, \beta_{s}, \gamma_{s}\right) d s\right. \\
& \left.+\Gamma_{0, T}^{\beta, \gamma} \widetilde{\mathcal{u}}\left(\zeta \frac{\widehat{L}(T)}{\Gamma_{0, T}^{\beta, \gamma}}\right)+\zeta x\right]=\inf _{\zeta>0}[\widetilde{V}(\zeta)+\zeta x] .
\end{aligned}
$$

Lemma 12. Under the assumptions of Lemma 11, the followings hold:

(i) $(\widehat{\beta}, \widehat{\gamma})$ attains the infimum in (39) with $\zeta=\widehat{\zeta}$.

(ii) The triple $(\widehat{\zeta}, \widehat{\beta}, \widehat{\gamma})$ attains the first infimum in (41).

(iii) The number $\widehat{\zeta} \in(0, \infty)$ attains the second infimum in (41).

(iv) There is no "duality gap" in (40); that is,

$$
\begin{aligned}
V_{*}(x) & =\bar{V}(x)=\underline{V}(x) \\
& =E\left[\int_{0}^{T} \Gamma_{0, s}^{\hat{\beta}, \widehat{\gamma}^{\prime}} F\left(s, \widehat{\beta}_{s}, \widehat{\gamma}_{s}\right) d s+\Gamma_{0, T}^{\hat{\beta}, \widehat{\gamma}} u(\widehat{\xi})\right] .
\end{aligned}
$$

Proof. (i) By (35) and (36),

$$
\begin{array}{r}
E\left[\int_{0}^{T} \Gamma_{0, s}^{\hat{\beta}, \widehat{\gamma}} F\left(s, \widehat{\beta}_{s}, \widehat{\gamma}_{s}\right) d s+\Gamma_{0, T}^{\hat{\beta}, \widehat{\mathcal{u}}}\left(\hat{\zeta} \frac{\widehat{L}(T)}{\Gamma_{0, T}^{\widehat{\beta}, \hat{\gamma}}}\right)\right] \\
=E\left[\int_{0}^{T} \Gamma_{0, s}^{\hat{\beta}, \widehat{\gamma}} F\left(s, \widehat{\beta}_{s}, \widehat{\gamma}_{s}\right) d s+\Gamma_{0, T}^{\hat{\beta}, \widehat{\gamma}} u(\widehat{\xi})\right]-\widehat{\zeta} x \\
\leq E\left[\int_{0}^{T} \Gamma_{0, s}^{\beta, \gamma} F\left(s, \beta_{s}, \gamma_{s}\right) d s+\Gamma_{0, T}^{\beta, \gamma} u(\widehat{\xi})\right]-\widehat{\zeta} x \\
\leq E\left[\int_{0}^{T} \Gamma_{0, s}^{\beta, \gamma} F\left(s, \beta_{s}, \gamma_{s}\right) d s+\Gamma_{0, T}^{\beta, \gamma} \widetilde{\mathcal{u}}\left(\widehat{\zeta} \frac{\widehat{L}(T)}{\Gamma_{0, T}^{\beta, \gamma}}\right)\right], \\
\forall(\beta, \gamma) \in \mathscr{B},
\end{array}
$$

where the last inequality is due to (33). 
(ii) By (35) and (36), we have

$$
\begin{aligned}
E & {\left[\int_{0}^{T} \Gamma_{0, s}^{\hat{\beta}, \widehat{\gamma}} F\left(s, \widehat{\beta}_{s}, \widehat{\gamma}_{s}\right) d s+\Gamma_{0, T}^{\hat{\beta}, \widehat{u}}\left(\widehat{\zeta} \frac{\widehat{L}(T)}{\Gamma_{0, T}^{\widehat{\beta}, \widehat{\gamma}}}\right)\right]+\widehat{\zeta} x } \\
= & E\left[\int_{0}^{T} \Gamma_{0, s}^{\hat{\beta}, \widehat{\gamma}} F\left(s, \widehat{\beta}_{s}, \widehat{\gamma}_{s}\right) d s+\Gamma_{0, T}^{\hat{\beta}, \widehat{\gamma}} u(\widehat{\xi})\right] \\
\leq & E\left[\int_{0}^{T} \Gamma_{0, s}^{\beta, \gamma} F\left(s, \beta_{s}, \gamma_{s}\right) d s+\Gamma_{0, T}^{\beta, \gamma} u(\widehat{\xi})\right] \\
\leq & E\left[\int_{0}^{T} \Gamma_{0, s}^{\beta, \gamma} F\left(s, \beta_{s}, \gamma_{s}\right) d s+\Gamma_{0, T}^{\beta, \gamma} \widetilde{\mathcal{u}}\left(\zeta \frac{\widehat{L}(T)}{\Gamma_{0, T}^{\beta, \gamma}}\right)\right] \\
& +\zeta x, \quad \forall(\beta, \gamma) \in \mathscr{B}, \forall \zeta \in(0, \infty)
\end{aligned}
$$

where the last inequality is an application of (33) to $\xi=\widehat{\xi}$.

(iii) By (i), (35), and (36),

$$
\begin{aligned}
\widetilde{V}(\widehat{\zeta})+ & \widehat{\zeta} x \\
=E & {\left[\int_{0}^{T} \Gamma_{0, s}^{\widehat{\beta}, \widehat{\gamma}} F\left(s, \widehat{\beta}_{s}, \widehat{\gamma}_{s}\right) d s+\Gamma_{0, T}^{\widehat{\beta}, \widehat{\mathcal{u}}}\left(\hat{\zeta} \frac{\widehat{L}(T)}{\Gamma_{0, T}^{\hat{\beta}, \hat{\gamma}}}\right)\right] } \\
& +\widehat{\zeta} x=E\left[\int_{0}^{T} \Gamma_{0, s}^{\widehat{\beta}, \widehat{\gamma}} F\left(s, \widehat{\beta}_{s}, \widehat{\gamma}_{s}\right) d s+\Gamma_{0, T}^{\widehat{\beta}, \widehat{\gamma}} u(\widehat{\xi})\right] \\
\leq & E\left[\int_{0}^{T} \Gamma_{0, s}^{\beta, \gamma} F\left(s, \beta_{s}, \gamma_{s}\right) d s+\Gamma_{0, T}^{\beta, \gamma} u(\widehat{\xi})\right] \\
\leq & {\left[\int_{0}^{T} \Gamma_{0, s}^{\beta, \gamma} F\left(s, \beta_{s}, \gamma_{s}\right) d s+\Gamma_{0, T}^{\beta, \gamma} \widetilde{\mathcal{u}}\left(\zeta \frac{\widehat{L}(T)}{\Gamma_{0, T}^{\beta, \gamma}}\right)\right] } \\
& +\zeta x, \quad \forall(\beta, \gamma) \in \mathscr{B}, \forall \zeta \in(0, \infty) .
\end{aligned}
$$

So we get $\widetilde{V}(\widehat{\zeta})+\widehat{\zeta} x \leq \inf _{(\beta, \gamma) \in \mathscr{B}} E\left[\int_{0}^{T} \Gamma_{0, s}^{\beta, \gamma} F\left(s, \beta_{s}, \gamma_{s}\right) d s+\right.$ $\left.\Gamma_{0, T}^{\beta, \gamma} \widetilde{u}\left(\zeta\left(\widehat{L}(T) / \Gamma_{0, T}^{\beta, \gamma}\right)\right)\right]+\zeta x=\widetilde{V}(\zeta)+\zeta x, \forall \zeta \in(0, \infty)$.

(iv) By (ii) and (35),

$$
V_{*}(x)
$$

$$
\begin{aligned}
= & E\left[\int_{0}^{T} \Gamma_{0, s}^{\hat{\beta}, \widehat{\gamma}} F\left(s, \widehat{\beta}_{s}, \widehat{\gamma}_{s}\right) d s+\Gamma_{0, T}^{\hat{\beta}, \widehat{\psi}} \widetilde{\mathcal{u}}\left(\frac{\widehat{L}(T)}{\Gamma_{0, T}^{\widehat{\beta}, \widehat{\gamma}}}\right)\right] \\
& +\widehat{\zeta} x=E\left[\int_{0}^{T} \Gamma_{0, s}^{\widehat{\beta}, \widehat{\gamma}} F\left(s, \widehat{\beta}_{s}, \widehat{\gamma}_{s}\right) d s+\Gamma_{0, T}^{\widehat{\beta}, \widehat{\gamma}} u(\widehat{\xi})\right] \\
= & \bar{V}(x)=\underline{V}(x) .
\end{aligned}
$$

This completes the proof.

In the following, we prove the existence of the quadruple $(\widehat{\xi}, \widehat{\beta}, \widehat{\gamma}, \widehat{\zeta})$ which is postulated in Lemma 11 .

Notice that the function $x \mapsto x \widetilde{u}(1 / x)$ is convex. By similar analysis as in Appendix B of [23], the following lemma holds.
Lemma 13. Under Assumptions 1, 7, and 8, for any given $\zeta>0$, there exists a pair $(\widehat{\beta}, \widehat{\gamma})=\left(\widehat{\beta}_{\zeta}, \widehat{\gamma}_{\zeta}\right) \in \mathscr{B}$ which attains the infimum in (39).

Lemma 14. Under Assumptions 1, 7, and 8, suppose

$$
E\left[\int_{0}^{T} \Gamma_{0, s}^{\beta, \gamma} F\left(s, \beta_{s}, \gamma_{s}\right) d s+\Gamma_{0, T}^{\beta, \gamma} \widetilde{u}\left(\zeta \frac{\widehat{L}(T)}{\Gamma_{0, T}^{\beta, \gamma}}\right)\right]<\infty,
$$

$$
\forall \zeta>0, \forall(\beta, \gamma) \in \mathscr{B} \text {. }
$$

Then for any given $x>0$, there exists a number $\widehat{\zeta}=\widehat{\zeta}_{x} \epsilon$ $(0, \infty)$ which attains $V_{*}(x)=\inf _{\zeta>0}[\widetilde{V}(\zeta)+\zeta x]$.

Proof.

Step 1. By the convexity of $\widetilde{u}$ and Lemma $13, \widetilde{V}(\cdot)$ is convex. Fix $\zeta>0$; denote $(\widehat{\beta}, \widehat{\gamma})=\left(\widehat{\beta}_{\zeta}, \widehat{\gamma}_{\zeta}\right)$ as in Lemma 13. For any $\delta>0$, we have

$$
\begin{aligned}
& \frac{\widetilde{V}(\zeta+\delta)-\widetilde{V}(\zeta)}{\delta} \\
& \leq \frac{\left.\left.E\left[\Gamma_{0, T}^{\hat{\beta}, \hat{\mathcal{u}}} \widetilde{u}(\zeta+\delta)\left(\widehat{L}(T) / \Gamma_{0, T}^{\hat{\beta}, \hat{\gamma}}\right)\right)-\Gamma_{0, T}^{\hat{\beta}, \widehat{\mathcal{u}}} \widetilde{\zeta}\left(\widehat{L}(T) / \Gamma_{0, T}^{\hat{\beta}, \widehat{\gamma}}\right)\right)\right]}{\delta} \\
& \leq E\left[\widehat{L}(T) \tilde{u}^{\prime}\left((\zeta+\delta)\left(\widehat{L}(T) / \Gamma_{0, T}^{\hat{\beta}, \hat{\gamma}}\right)\right)\right] \\
& =-E\left[\widehat{L}(T) I\left((\zeta+\delta)\left(\widehat{L}(T) / \Gamma_{0, T}^{\widehat{\beta}, \hat{\gamma}}\right)\right)\right] .
\end{aligned}
$$

Then, by Levi's lemma,

$$
\begin{aligned}
& \lim _{\delta \rightarrow 0+} \frac{\widetilde{V}(\zeta+\delta)-\widetilde{V}(\zeta)}{\delta} \leq-E\left[\widehat{L}(T) I\left(\zeta \frac{\widehat{L}(T)}{\Gamma_{0, T}^{\widehat{\beta}, \widehat{\gamma}}}\right)\right], \\
& \lim _{\delta \rightarrow 0+} \frac{\widetilde{V}(\zeta)-\widetilde{V}(\zeta-\delta)}{\delta} \geq-E\left[\widehat{L}(T) I\left(\zeta \frac{\widehat{L}(T)}{\Gamma_{0, T}^{\hat{\beta}, \hat{\gamma}}}\right)\right] .
\end{aligned}
$$

Since $\widetilde{V}(\cdot)$ is convex, we obtain that $\widetilde{V}(\cdot)$ is differentiable on $(0, \infty)$ and $\widetilde{V}^{\prime}(\zeta)=-E\left[\widehat{L}(T) I\left(\zeta\left(\widehat{L}(T) / \Gamma_{0, T}^{\widehat{\beta}, \widehat{\gamma}}\right)\right)\right]$.

Step 2. Because $\mu(\cdot)$ is bounded, we have that, for any $\zeta \in$ $(0, \infty), \widehat{L}(T) / \Gamma_{0, T}^{\widehat{\beta}, \widehat{\gamma}}<+\infty$, a.s. Then,

$$
\begin{aligned}
\widetilde{V}^{\prime}(+\infty) & :=\lim _{\zeta \rightarrow+\infty} \widetilde{V}^{\prime}(\zeta) \\
& =-\lim _{\zeta \rightarrow \infty} E\left[\widehat{L}(T) I\left(\zeta \frac{\widehat{L}(T)}{\Gamma_{0, T}^{\widehat{\beta}, \hat{\gamma}}}\right)\right]=0, \\
\widetilde{V}^{\prime}(0) & :=\lim _{\zeta \rightarrow 0+} \widetilde{V}^{\prime}(\zeta) \\
& =-\lim _{\zeta \rightarrow 0+} E\left[\widehat{L}(T) I\left(\zeta \frac{\widehat{L}(T)}{\Gamma_{0, T}^{\widehat{\beta}, \widehat{\gamma}}}\right)\right]=-\infty
\end{aligned}
$$


Thus, there exists a number $\widehat{\zeta}$ which attains $V_{*}(x)$ and $\widetilde{V}^{\prime}(\widehat{\zeta})=-x \in(-\infty, 0)$. This completes the proof.

Lemma 15. Under Assumptions 1, 7, and 8, $V_{*}(x)=$ $E\left[\int_{0}^{T} \Gamma_{0, s}^{\hat{\beta}, \widehat{\gamma}} F\left(s, \widehat{\beta}_{s}, \widehat{\gamma}_{s}\right) d s+\Gamma_{0, T}^{\widehat{\beta}, \widehat{\gamma}} u\left(\widehat{\zeta}\left(\widehat{L}(T) / \Gamma_{0, T}^{\widehat{\beta}, \widehat{\gamma}}\right)\right)\right]+\widehat{\zeta} x$ with $\widehat{\zeta}=\widehat{\zeta}_{x}$ as in Lemma 14 and $(\widehat{\beta}, \widehat{\gamma})=\left(\widehat{\beta}_{\widehat{\zeta}}, \widehat{\gamma}_{\tilde{\zeta}}\right)$ as in Lemma 13.

Proof. We have

$$
\begin{aligned}
& E\left[\int_{0}^{T} \Gamma_{0, s}^{\hat{\beta}, \widehat{\gamma}} F\left(s, \widehat{\beta}_{s}, \widehat{\gamma}_{s}\right) d s+\Gamma_{0, T}^{\widehat{\beta}, \widehat{u}} \widetilde{\zeta}\left(\widehat{\zeta} \frac{\widehat{L}(T)}{\Gamma_{0, T}^{\widehat{\beta}, \widehat{\gamma}}}\right)\right]+\widehat{\zeta} x \\
& =\widetilde{V}(\widehat{\zeta})+\widehat{\zeta} x \leq \widetilde{V}(\zeta)+\zeta x \\
& \quad \leq E\left[\int_{0}^{T} \Gamma_{0, s}^{\beta, \gamma} F\left(s, \beta_{s}, \gamma_{s}\right) d s+\Gamma_{0, T}^{\beta, \gamma} \widetilde{u}\left(\zeta \frac{\widehat{L}(T)}{\Gamma_{0, T}^{\beta, \gamma}}\right)\right. \\
& \quad+\zeta x], \quad \forall(\beta, \gamma) \in \mathscr{B}, \forall \zeta \in(0, \infty), \forall x>0 .
\end{aligned}
$$

This completes the proof.

Our main result is the following theorem.

Theorem 16. Under Assumptions 1, 7, and 8 , let $(\widehat{\zeta}, \widehat{\beta}, \widehat{\gamma})$ as in Lemma 15 and define $\widehat{\xi}=I\left(\widehat{\zeta}\left(\widehat{L}(T) / \Gamma_{0, T}^{\widehat{\beta}, \widehat{\gamma}}\right)\right)$ a.s. If $\widehat{\xi} \in U$, then $(\widehat{\zeta}, \widehat{\beta}, \widehat{\gamma}, \widehat{\xi})$ satisfies all the conditions in Lemma 11, that is, (34) and (36).

Proof. Notice that

$$
\begin{aligned}
& \widetilde{V}(\widehat{\zeta})=\inf _{(\beta, \gamma) \in \mathscr{B}} E\left[\int_{0}^{T} \Gamma_{0, s}^{\beta, \gamma} F\left(s, \beta_{s}, \gamma_{s}\right) d s\right. \\
& \left.+\Gamma_{0, T}^{\beta, \gamma} \widetilde{u}\left(\widehat{\zeta} \frac{\widehat{L}(T)}{\Gamma_{0, T}^{\beta, \gamma}}\right)\right]=E\left[\int_{0}^{T} \Gamma_{0, s}^{\hat{\beta}, \widehat{\gamma}} F\left(s, \widehat{\beta}_{s}, \widehat{\gamma}_{s}\right) d s\right. \\
& \left.+\Gamma_{0, T}^{\hat{\beta}, \widehat{\gamma}} \widetilde{u}\left(\widehat{\zeta} \frac{\widehat{L}(T)}{\Gamma_{0, T}^{\widehat{\beta}, \hat{\gamma}}}\right)\right] .
\end{aligned}
$$

Applying the maximum principle in Peng [24], we obtain a necessary condition for $(\widehat{\beta}, \widehat{\gamma})$ :

$$
\begin{array}{r}
F\left(t, \beta_{t}, \gamma_{t}\right)+p_{t} \beta_{t}+q_{t} \gamma_{t} \geq F\left(t, \widehat{\beta}_{t}, \widehat{\gamma}_{t}\right)+p_{t} \widehat{\beta}_{t}+q_{t} \widehat{\gamma}_{t}, \\
\forall(\beta, \gamma) \in \mathscr{B},
\end{array}
$$

where $\left(p_{t}, q_{t}\right)$ is the solution of the adjoint equation

$$
\begin{aligned}
-d p_{t} & =\left(F\left(t, \widehat{\beta}_{t}, \widehat{\gamma}_{t}\right)+p_{t} \widehat{\beta}_{t}+q_{t}^{\prime} \widehat{\gamma}_{t}\right) d t-q_{t}^{\prime} d \widehat{W}(t), \\
p_{T} & =u\left(I\left(\widehat{\zeta} \frac{\widehat{L}(T)}{\Gamma_{0, T}^{\widehat{\beta}, \hat{\gamma}}}\right)\right) .
\end{aligned}
$$

$\forall(\beta, \gamma) \in \mathscr{B}$; let $\left(y_{t}, z_{t}\right)$ and $\left(\tilde{y}_{t}, \tilde{z}_{t}\right)$ be the unique solutions of the following two linear BSDEs:

$$
\begin{aligned}
y_{t}= & u(\widehat{\xi})+\int_{t}^{T}\left(y_{s} \widehat{\beta}_{s}+z_{s}^{\prime} \hat{\gamma}_{s}+F\left(s, \widehat{\beta}_{s}, \widehat{\gamma}_{s}\right)\right) d s \\
& -\int_{t}^{T} z_{s}^{\prime} d \widehat{W}(s), \\
\tilde{y}_{t}= & u(\widehat{\xi})+\int_{t}^{T}\left(\tilde{y}_{s} \beta_{s}+\widetilde{z}_{s}^{\prime} \gamma_{s}+F\left(s, \beta_{s}, \gamma_{s}\right)\right) d s \\
& -\int_{t}^{T} \widetilde{z}_{s}^{\prime} d \widehat{W}(s) .
\end{aligned}
$$

By (53) and the comparison theorem of BSDE, we have $y_{t} \leq \tilde{y}_{t}, t \in[0, T]$, a.s., especially $y_{0} \leq \tilde{y}_{0}$.

Solving the above linear BSDEs gives

$$
\begin{aligned}
& y_{0}=E\left[\int_{0}^{T} \Gamma_{0, s}^{\widehat{\beta}, \widehat{\gamma}} F\left(s, \widehat{\beta}_{s}, \widehat{\gamma}_{s}\right) d s+\Gamma_{0, T}^{\widehat{\beta}, \widehat{\gamma}} u(\widehat{\xi})\right], \\
& \tilde{y}_{0}=E\left[\int_{0}^{T} \Gamma_{0, s}^{\beta, \gamma} F\left(s, \beta_{s}, \gamma_{s}\right) d s+\Gamma_{0, T}^{\beta, \gamma} u(\widehat{\xi})\right] .
\end{aligned}
$$

So

$$
\begin{aligned}
& E\left[\int_{0}^{T} \Gamma_{0, s}^{\widehat{\beta}, \hat{\gamma}} F\left(s, \widehat{\beta}_{s}, \widehat{\gamma}_{s}\right) d s+\Gamma_{0, T}^{\widehat{\beta}, \widehat{\gamma}} u(\widehat{\xi})\right] \\
& \leq E\left[\int_{0}^{T} \Gamma_{0, s}^{\beta, \gamma} F\left(s, \beta_{s}, \gamma_{s}\right) d s+\Gamma_{0, T}^{\beta, \gamma} u(\widehat{\xi})\right], \\
& \forall(\beta, \gamma) \in \mathscr{B},
\end{aligned}
$$

which exactly is (36).

By Lemma $14, \widetilde{V}^{\prime}(\widehat{\zeta})=-x$. By Lemma 13 ,

$$
\begin{aligned}
& \widetilde{V}(\widehat{\zeta}) \\
& =E\left[\int_{0}^{T} \Gamma_{0, s}^{\widehat{\beta}, \widehat{\gamma}} F\left(s, \widehat{\beta}_{s}, \widehat{\gamma}_{s}\right) d s+\Gamma_{0, T}^{\widehat{\beta}, \widehat{\mathcal{u}}} \widetilde{\zeta}\left(\frac{\widehat{L}(T)}{\Gamma_{0, T}^{\widehat{\beta}, \widehat{\gamma}}}\right)\right] .
\end{aligned}
$$

Differentiating both sides of (59) as functions of $\widehat{\zeta}$, we get

$$
E\left[I\left(\widehat{\zeta} \frac{\widehat{L}(T)}{\Gamma_{0, T}^{\widehat{\beta}, \widehat{r}}}\right) \widehat{L}(T)\right]=x .
$$

This completes the proof.

Remark 17. It is worth pointing out that the adjoint process $p_{t}$ in the proof of the above theorem coincides with the optimal utility process $y_{t}$ in (55).

\section{4. $K$-Ignorance}

In this section, we study a special case which is called $K$-ignorance by Chen and Epstein [25]. In this case, the generator $f$ is specified as

$$
f(t, y, z)=-K|z|, \quad K \geq 0 .
$$


Chen and Epstein interpreted the term $K|z|$ as modeling ambiguity aversion rather than risk aversion. $f(z)=-K|z|$ is not differentiable. But it is concave and $f(z)=\inf _{|\gamma| \leq K}(\gamma z)$. Then our results in the above section are still applicable.

In this section, we assume $d=1, \sigma \equiv 1$. The wealth equation and recursive utility become

$$
\begin{aligned}
-d X(t) & =-q^{\prime}(t) \widehat{\mu}(t) d t-q^{\prime}(t) d \widehat{W}(t), \\
X(T) & =\xi, \\
-d Y(t) & =-K|Z(t)| d t-Z^{\prime}(t) d \widehat{W}(t), \\
Y(T) & =u(\xi) .
\end{aligned}
$$

Our problem is formulated as follows:

$$
\begin{aligned}
\text { Maximize } & J(\xi):=Y_{0}^{\xi}, \\
\text { s.t. } \quad & \xi \in U, \\
& X(0)=x, \\
& (X(\cdot), q(\cdot)),(Y(\cdot), Z(\cdot)) \text { satisfies Eq. (62). }
\end{aligned}
$$

Now Lemma 9 can be simplified to the following lemma.

Lemma 18. For $\xi \in U$, the solutions $(X(\cdot), q(\cdot))$ and $(Y(\cdot), Z(\cdot))$ of $(62)$ can be represented as

$$
\begin{aligned}
& X(t)=\widehat{L}^{-1}(t) E\left[\widehat{L}(T) \xi \mid \mathscr{G}_{t}\right], \\
& Y(t)=\text { ess } \inf _{\gamma \in \mathscr{B}}\left(\Gamma_{0, t}^{0, \gamma}\right)^{-1}(t) E\left[\Gamma_{0, T}^{0, \gamma} u(\xi) \mid \mathscr{G}_{t}\right],
\end{aligned}
$$

where

$$
\begin{aligned}
\widehat{L}(t) & =e^{-\int_{0}^{t} \widehat{\mu}(s) d \widehat{W}(s)-(1 / 2) \int_{0}^{t}|\widehat{\mu}(s)|^{2} d s}, \\
\Gamma_{0, t}^{0, \gamma} & =e^{\int_{0}^{t} \gamma_{s} d \widehat{W}(s)-(1 / 2) \int_{0}^{t}\left|\gamma_{s}\right|^{2} d s,} \\
\mathscr{B} & =\left\{\gamma=\left\{\gamma_{t}\right\}_{t \geq 0} \mid \gamma_{t}\right. \text { is } \\
\cdot & \mathbb{G}-\text { progressively measurable, }\left|\gamma_{t}\right| \leq K, t \\
\in & {[0, T], \text { a.s. }\} . }
\end{aligned}
$$

For any $\gamma \in \mathscr{B}, \Gamma_{0, t}^{0, \gamma}$ is $(\mathbb{G}, P)$-martingale. Then, a new probability measure $P_{\gamma}$ is defined on $\mathscr{G}_{T}$ by

$$
\frac{d P_{\gamma}}{d P}=\Gamma_{0, T}^{0, \gamma}
$$

and $\widehat{W}_{\gamma}(t)=\widehat{W}(t)-\int_{0}^{t} \gamma_{s} d s$ is a Brownian motion under $P_{\gamma}$. Thus, $Y(0)=\inf _{\gamma \in \mathscr{B}} E_{\gamma}[u(\xi)]$ where $E_{\gamma}[\cdot]$ is the expectation operator with respect to $P_{\gamma}$.

Our problem (63) is equivalent to the following problem:

$$
\begin{array}{cl}
\text { Maximize } & J(\xi)=\inf _{\gamma \in \mathscr{B}} E_{\gamma} u(\xi) \\
\text { s.t. } & \xi \in \mathscr{A}(x) .
\end{array}
$$

The auxiliary dual problem in (39) becomes

$$
\widetilde{V}(\zeta) \equiv \widetilde{V}(\zeta ; x):=\inf _{\gamma \in \mathscr{B}} E_{\gamma} \widetilde{u}\left(\zeta Z_{\gamma}(T)\right), \quad 0<\zeta<\infty,
$$

where $Z_{\gamma}(t):=\widehat{L}(t) / \Gamma_{0, t}^{0, \gamma}, t \in[0, T]$ a.s. and

$$
\begin{aligned}
V_{*}(x) & :=\inf _{\zeta>0, \gamma \in \mathscr{B}}\left[E_{\gamma} \widetilde{u}\left(\zeta Z_{\gamma}(T)\right)+\zeta x\right] \\
& =\inf _{\zeta>0}[\widetilde{V}(\zeta)+\zeta x] .
\end{aligned}
$$

Applying the procedure in the previous section, we can find the saddle point. So we list the results without proof except Lemma 19 in which a new proof is given.

Lemma 19. Under Assumption 8, for any given $\zeta>0$, there exists a unique $\widehat{\gamma}=\widehat{\gamma}_{\zeta} \in \mathscr{B}$ which attains the infimum in (68).

Proof. Set $\mathscr{B}^{\prime}=\left\{\Gamma_{0, T}^{0, \gamma} \mid \gamma \in \mathscr{B}\right\}, \mathscr{M}=\left\{M_{\gamma}(T):=\Gamma_{0, T}^{0, \gamma} / \widehat{L}(T) \mid\right.$ $\gamma \in \mathscr{B}\}$, and $g(x)=x \tilde{u}(\zeta / x)$ for $x>0$. Then problem (68) becomes

$$
\begin{aligned}
\widetilde{V}(\zeta) & =\inf _{M_{\gamma}(T) \in \mathscr{M}} \widetilde{E}\left[M_{\gamma}(T) \widetilde{u}\left(\zeta \frac{1}{M_{\gamma}(T)}\right)\right] \\
& =\inf _{M_{\gamma}(T) \in \mathscr{M}} \widetilde{E}\left[g\left(M_{\gamma}(T)\right)\right],
\end{aligned}
$$

where $\widetilde{E}[\cdot]$ is the expectation operator with respect to the risk neutral measure $\widetilde{P}$. By Theorem 2.1 in [25], we know $\mathscr{B}^{\prime}$ is norm closed in $L^{1}(\Omega)$. So $\mathscr{B}$ is closed under a.s. convergence because $B$ is uniformly integrable. As a consequence, $\mathscr{M}$ is closed under a.s. convergence.

Consider a minimizing sequence $\left\{M_{\gamma^{n}}(T)\right\}_{n \geq 1}$ for (70); that is

$$
\lim _{n \rightarrow \infty} \widetilde{E}\left[g\left(M_{\gamma^{n}}(T)\right)\right]=\widetilde{V}(\zeta)
$$

By Komlos' theorem, there exists a sequence $\bar{M}_{\gamma^{n}}(T) \epsilon$ $\operatorname{conv}\left(M_{\gamma^{n}}(T), M_{\gamma^{n+1}}(T), \ldots,\right)$; that is, $\bar{M}_{\gamma^{n}}(T)=$ $\sum_{k=n}^{T_{n}} \lambda_{k} M_{\gamma^{k}}(T), \lambda_{k} \in[0,1]$, and $\sum_{k=n}^{T_{n}} \lambda_{k}=1$, such that the sequence $\left\{M_{\gamma^{n}}(T)\right\}_{n \geq 1}$ converges a.s. to a random variable $M$. By a.s. closedness of $\mathscr{M}$, we have $M \in \mathscr{M}$; that is, $\exists \widehat{\gamma} \in \mathscr{B}$, s.t. $M=M_{\widehat{\gamma}}(T)$. Note that $g$ is a strictly convex continuous function; we have

$$
\begin{aligned}
\widetilde{E}[g(M)] & =\widetilde{E}\left[\lim _{n \rightarrow \infty} g\left(\bar{M}_{\gamma^{n}}(T)\right)\right] \\
& \leq \liminf _{n \rightarrow \infty} \widetilde{E}\left[g\left(\bar{M}_{\gamma^{n}}(T)\right)\right] \\
& \leq \liminf _{n \rightarrow \infty} \lambda_{k} \sum_{k=n}^{T_{n}} \widetilde{E}\left[g\left(M_{\gamma^{k}}(T)\right)\right] \\
& =\liminf _{n \rightarrow \infty} \widetilde{E}\left[g\left(M_{\gamma^{n}}(T)\right)\right]=\widetilde{V}(\zeta) .
\end{aligned}
$$

The uniqueness follows from the strict convexity of $g$. This completes the proof. 
Lemma 20. Under Assumption 8, if $\widetilde{E}\left[I\left(\zeta Z_{\gamma}(T)\right)\right]<\infty$, $\forall \zeta>0, \forall \gamma \in \mathscr{B}$, then for any given $x>0$, there exists a number $\widehat{\zeta}=\widehat{\zeta}_{x} \in(0, \infty)$ which attains the infimum of $V_{*}(x)=\inf _{\zeta>0}[\widetilde{V}(\zeta)+\zeta x]$.

Lemma 21. Under Assumption 8, $V_{*}(x)=E_{\widehat{\gamma}} \widetilde{u}\left(\widehat{\zeta} Z_{\widehat{\gamma}}(T)\right)+\widehat{\zeta} x$ with $\widehat{\zeta}=\widehat{\zeta}_{x}$ as in Lemma 20 and $\widehat{\gamma}=\widehat{\gamma}_{\widehat{\zeta}}$ as in Lemma 19.

Theorem 22. Under Assumption 8, let $(\widehat{\zeta}, \widehat{\gamma})$ be the same as in Lemma 21; then the optimal terminal wealth of problem (67) is

$$
\widehat{\xi}=I\left(\widehat{\zeta} Z_{\widehat{\gamma}}(T)\right), \quad \text { a.s. }
$$

\section{if $\widehat{\xi}$ belongs to $U$.}

In the following, we give some examples to illustrate our above analysis.

Example 23 (constant absolute risk aversion). Suppose that $u(x)=1-e^{-\alpha x}, x \in \mathbb{R}, \alpha>0$, and the wealth of the investor may be negative. This utility function $u$ does not satisfy Assumption 8. But it satisfies the following assumption.

Assumption 24. $u$ is strictly increasing, strictly concave, and continuously differentiable, and

$$
\begin{aligned}
u^{\prime}(-\infty) & :=\lim _{x \downarrow-\infty} u^{\prime}(x)=\infty, \\
u^{\prime}(\infty) & :=\lim _{x \rightarrow \infty} u^{\prime}(x)=0 .
\end{aligned}
$$

Note that under Assumption 24, the wealth process of the investor is allowed to be negative; the results in this section still hold.

For this example, $I(\zeta)=-(1 / \alpha) \ln (\zeta / \alpha), \zeta>0$, and $\widetilde{u}(\zeta)=$ $1-\zeta / \alpha+(\zeta / \alpha) \ln (\zeta / \alpha), \zeta>0$. Then the value function of the auxiliary dual problem (68) is

$$
\begin{aligned}
E_{\gamma} \widetilde{u}\left(\zeta Z_{\gamma}(T)\right)= & 1-\frac{\zeta}{\alpha}+\frac{\zeta}{\alpha} \ln \frac{\zeta}{\alpha}+\frac{\zeta}{\alpha} \widetilde{E}\left[\ln Z_{\gamma}(T)\right] \\
= & 1-\frac{\zeta}{\alpha}+\frac{\zeta}{\alpha} \ln \frac{\zeta}{\alpha} \\
& +\frac{\zeta}{2 \alpha} \widetilde{E} \int_{0}^{T}\left(\widehat{\mu}(t)+\gamma_{t}\right)^{2} d t, \quad \zeta>0 .
\end{aligned}
$$

Apparently, $\hat{\gamma}_{t}$ (optimal $\gamma_{t}$ ) which attains the infimum of Problem (68) is independent of $\zeta$. It is easy to see that

$$
\widehat{\gamma}_{t}=((-K) \vee(-\widehat{\mu}(t))) \wedge K, \quad t \in[0, T] \text {, a.s. }
$$

The optimal value of problem (68) is

$$
\widetilde{V}(\zeta)=1-\frac{\zeta}{\alpha}+\frac{\zeta}{\alpha} \ln \frac{\zeta}{\alpha}+\frac{\zeta}{2 \alpha} \widetilde{E} \int_{0}^{T}\left(\widehat{\mu}(t)+\widehat{\gamma}_{t}\right)^{2} d t,
$$

and the Lagrange multiplier in Lemma 20 is

$$
\begin{aligned}
\widehat{\zeta} & \equiv \widehat{\zeta}_{x}=\alpha e^{-(1 / 2) \widetilde{E} \int_{0}^{T}\left(\widehat{\mu}(t)+\widehat{\psi}_{t}\right)^{2} d t-\alpha x} \\
& =\arg \min _{\zeta>0}[\widetilde{V}(\zeta)+\zeta x] .
\end{aligned}
$$

Thus, the optimal terminal wealth in Theorem 22 is

$$
\widehat{\xi}=-\frac{1}{\alpha} \ln \frac{\widehat{\zeta} Z_{\widehat{\gamma}}(T)}{\alpha} .
$$

Moreover, it is easy to check that $(Y(t), Z(t)):=(1-$ $\left.(\widehat{\zeta} / \alpha) Z_{\widehat{\gamma}}(t),(\widehat{\zeta} / \alpha)\left(\widehat{\mu}(t)+\widehat{\gamma}_{t}\right) Z_{\widehat{\gamma}}(t)\right)$ and $t \in[0, T]$ uniquely solves the utility equation in (62) when $\xi=\widehat{\xi}$.

Note that $\widehat{\mu}(t)$ and $\hat{\gamma}_{t}$ are bounded; it is easy to verify $\widehat{\xi} \in$ $L_{\mathscr{G}_{T}}^{2}$ and $u(\widehat{\xi}) \in L_{\mathscr{G}_{T}}^{2}$.

Example 25 (logarithmic utility function). Suppose $u(x)=$ $\ln x, x>0$. In this case,

$$
\begin{aligned}
I(\zeta) & =\frac{1}{\zeta}, \\
\widetilde{u}(\zeta) & =-\ln \zeta-1,
\end{aligned}
$$

$$
\zeta>0 .
$$

Then the value function of the auxiliary dual problem (68) is

$$
\begin{aligned}
E_{\gamma} \widetilde{u}\left(\zeta Z_{\gamma}(T)\right) & =E_{\gamma}\left[-\ln \left(\zeta Z_{\gamma}(T)\right)-1\right] \\
& =E_{\gamma}\left[-\ln Z_{\gamma}(T)\right]-\ln \zeta-1 \\
& =\frac{1}{2} E_{\gamma} \int_{0}^{T}\left(\widehat{\mu}(t)+\gamma_{t}\right)^{2} d t-\ln \zeta-1,
\end{aligned}
$$

$$
\zeta>0
$$

So the optimal $\widehat{\gamma}_{t}$ is independent of $\zeta$. Consider the following BSDE:

$$
\begin{aligned}
y_{\gamma}(t)= & E_{\gamma}\left[\int_{t}^{T}\left(\widehat{\mu}(s)+\gamma_{s}\right)^{2} d s \mid \mathscr{G}_{t}\right] \\
= & \int_{t}^{T}\left[\left(\widehat{\mu}(s)+\gamma_{s}\right)^{2}+\gamma_{s} z_{\gamma}(s)\right] d s \\
& -\int_{t}^{T} z_{\gamma}(s) d \widehat{W}(s) .
\end{aligned}
$$

Set

$$
f\left(t, z_{t}\right)=\inf _{\gamma \in \mathscr{B}}\left[\left(\widehat{\mu}(t)+\gamma_{t}\right)^{2}+\gamma_{t} z_{t}\right]= \begin{cases}K^{2}-2 K \widehat{\mu}(t)-K z_{t}+\widehat{\mu}(t)^{2}, & \text { if }-2 \widehat{\mu}(t)+2 K<z_{t} ; \\ -\frac{1}{4} z_{t}^{2}-\widehat{\mu}(t) z_{t}, & \text { if }-2 \widehat{\mu}(t)-2 K \leq z_{t} \leq-2 \widehat{\mu}(t)+2 K ; \\ K^{2}+2 K \widehat{\mu}(t)+K z_{t}+\widehat{\mu}(t)^{2}, & \text { if } z_{t}<-2 \widehat{\mu}(t)-2 K, t \in[0, T], \text { a.s. }\end{cases}
$$


It is easy to show that $f\left(t, z_{t}\right)$ is uniformly Lipschitz, so the following BSDE has a unique solution which we still denoted by $\left(y_{t}, z_{t}\right)$.

$$
y_{t}=\int_{t}^{T} f\left(s, z_{s}\right) d s-\int_{t}^{T} z_{s} d \widehat{W}(s)
$$

Then the infimum in problem (68) is attained at

$$
\begin{aligned}
\widehat{\gamma}_{t}= & \arg \inf _{\gamma \in \mathscr{B}}\left[\left(\widehat{\mu}(t)+\gamma_{t}\right)^{2}+\gamma_{t} z_{t}\right] \\
= & -K I_{\left\{-2 \widehat{\mu}(t)+2 K<z_{t}\right\}} \\
& +\left(-\widehat{\mu}(t)-\frac{z_{t}}{2}\right) I_{\left\{-2 \widehat{\mu}(t)-2 K \leq z_{t} \leq-2 \widehat{\mu}(t)+2 K\right\}} \\
& +K I_{\left\{z_{t}<-2 \widehat{\mu}(t)-2 K\right\}}, \quad t \in[0, T], \text { a.s. }
\end{aligned}
$$

The Lagrange multiplier in Lemma 20 is

$$
\widehat{\zeta} \equiv \widehat{\zeta}_{x}=\frac{1}{x}=\arg \min _{\zeta>0}[\widetilde{V}(\zeta)+\zeta x]
$$

The optimal terminal wealth in Theorem 22 is

$$
\widehat{\xi}=\frac{x}{Z_{\widehat{\gamma}}(T)} .
$$

Note that $\widehat{\mu}(t)$ and $\widehat{\gamma}_{t}$ are bounded; it is easy to verify $\widehat{\xi} \in$ $L_{\mathscr{G}_{T}}^{2}$ and $u(\widehat{\xi}) \in L_{\mathscr{G}_{T}}^{2}$.

Example 26. Suppose that the appreciation rate $\mu(t)$ is a bounded deterministic function of $t$. In this case, $\mathscr{G}_{t}=\mathscr{F}_{t}$, $t \geq 0$, and we claim that

$$
\widehat{\gamma}_{t}=((-K) \vee(-\mu(t))) \wedge K, \quad t \in[0, T] .
$$

Proof. We show that $\widehat{\gamma}$ defined above attains the infimum of (68). Denote

$$
v(t, x) \equiv v(t, x ; \zeta):=\widetilde{E}\left[g\left(x \frac{M_{\widehat{\gamma}}(T)}{M_{\widehat{\gamma}}(t)}\right)\right] .
$$

Then $v(t, x)$ is the solution of the partial differential equation $\partial v / \partial t+(1 / 2)\left(\partial^{2} v / \partial x^{2}\right) x^{2}\left(\mu_{t}+\widehat{\gamma}_{t}\right)^{2}=0$.

$\forall \gamma \in \mathscr{B}$; applying Itô's formula to $v\left(t, M_{\gamma}(t)\right)$, we have

$$
\begin{aligned}
d v\left(t, M_{\gamma}(t)\right) & \\
= & {\left[\frac{\partial v}{\partial t}+\frac{1}{2} \frac{\partial^{2} v}{\partial x^{2}}\left(M_{\gamma}(t)\right)^{2}\left(\mu(t)+\gamma_{t}\right)^{2}\right] d t } \\
& +\frac{\partial v}{\partial x} M_{\gamma}(t)\left(\mu(t)+\gamma_{t}\right) d \widetilde{W}(t) \\
= & \frac{1}{2} \frac{\partial^{2} v}{\partial x^{2}}\left(M_{\gamma}(t)\right)^{2}\left[\left(\mu(t)+\gamma_{t}\right)^{2}-\left(\mu(t)+\widehat{\gamma}_{t}\right)^{2}\right] d t \\
& +\frac{\partial v}{\partial x} M_{\gamma}(t)\left(\mu(t)+\gamma_{t}\right) d \widetilde{W}(t) .
\end{aligned}
$$

By the definition of $\widehat{\gamma}_{t}(88)$, we have $\left(\mu(t)+\gamma_{t}\right)^{2}-(\mu(t)+$ $\left.\widehat{\gamma}_{t}\right)^{2} \geq 0, t \in[0, T]$. The convexity of $v(t, \cdot)$ guarantees that $v\left(t, M_{\gamma}(t)\right)$ is a submartingale. Thus, $\forall \gamma \in \mathscr{B}$,

$$
\begin{aligned}
E_{\gamma}\left[\widetilde{u}\left(\zeta Z_{\gamma}(T)\right)\right] & =\widetilde{E}\left[g\left(M_{\gamma}(T)\right)\right]=\widetilde{E} v\left(T, M_{\gamma}(T)\right) \\
& \geq \widetilde{E} v\left(0, M_{\gamma}(0)\right)=\widetilde{E}\left[g\left(M_{\widehat{\gamma}}(T)\right)\right] \\
& =E_{\widehat{\gamma}}\left[\widetilde{u}\left(\zeta Z_{\widehat{\gamma}}(T)\right)\right] .
\end{aligned}
$$

This completes the proof.

Example 27. Suppose that $|\mu(\cdot)| \leq K$, a.e., a.s. Then we have

$$
\widehat{\gamma}_{t}=-\widehat{\mu}(t), \quad t \in[0, T] \text {, a.s. }
$$

Note that $\mu$ belongs to $\mathscr{B}$ when $|\mu(\cdot)| \leq K$, a.e. a.s. Due to the convexity of $g$, we have that $\forall \gamma \in \mathscr{B}$,

$$
\begin{aligned}
\widetilde{E}\left[g\left(M_{\gamma}(t)\right)\right] & \geq g\left(\widetilde{E}\left(M_{\gamma}(T)\right)\right)=g(1) \\
& \equiv g\left(M_{\widehat{\gamma}}(T)\right) \equiv \widetilde{E}\left[g\left(M_{\widehat{\gamma}}(T)\right)\right] .
\end{aligned}
$$

In this case, $P_{\widehat{\gamma}}$ coincides with the risk neutral probability $\widetilde{P}$ on $\mathscr{G}_{T}$ which leads to the optimal terminal wealth $\widehat{\xi}=x$. This means that the investor will not invest in the risk assets at all.

Remark 28. For recursive utility for consumption of the form

$$
\begin{aligned}
Y(t)= & u(X(T))+\int_{t}^{T}\left(u\left(c_{s}\right)+f(s, Y(s), Z(s))\right) d s \\
& -\int_{t}^{T} Z(s) d \widehat{W}(s),
\end{aligned}
$$

where $u$ and $f$ satisfy Assumptions 2 and 8 and Assumptions 1 and 7, respectively, our method still works well.

\section{Terminal Perturbation Method}

When the generator of the recursive utility (4) is nonconcave, the dual method is not applicable. In this case, we apply the terminal perturbation method to obtain a characterization of the optimal terminal wealth. We need the following smooth assumption.

Assumption 29. $f$ is continuously differentiable in $(y, z)$.

Let $\xi^{*}$ be an optimal terminal wealth for (19); that is,

$$
Y^{\xi^{*}}(0)=\sup _{\xi \in \mathscr{A}(x)} Y^{\xi}(0)
$$

And let $\left(X^{*}(\cdot), q^{*}(\cdot), Y^{*}(\cdot), Z^{*}(\cdot)\right)$ be the corresponding state processes of (17).

Set

$$
\bar{\Omega}:=\left\{\omega \in \Omega \mid \xi^{*}(\omega)=0\right\} .
$$

By the terminal perturbation method in $[17,18]$, we have the following stochastic maximum principle. 
Theorem 30. Under Assumptions 1, 2, and 29, if $\xi^{*}$ is the optimal wealth of problem (19), then there exist $h_{0} \in \mathbb{R}, h_{1} \geq 0$, and $\left|h_{0}\right|^{2}+h_{1}^{2}=1$ such that

$$
\begin{aligned}
& h_{0} m(T)+h_{1} u^{\prime}\left(\xi^{*}\right) n(T) \geq 0 \text {, a.s. on } \bar{\Omega} ; \\
& h_{0} m(T)+h_{1} u^{\prime}\left(\xi^{*}\right) n(T)=0 \text {, a.s. on } \bar{\Omega}^{c} \text {, }
\end{aligned}
$$

where

$$
\begin{aligned}
& \begin{array}{l}
d m(t)=-\widehat{\mu}^{\prime}(t) \sigma^{\prime-1}(t) m(t) d \widehat{W}(t), \quad m(0)=1 ; \\
\qquad n(t)=f_{Y}^{*}(t) n(t) d t+f_{Z}^{* \prime}(t) n(t) d \widehat{W}(t), \\
\qquad n(0)=1,
\end{array} \\
& \text { and } f_{Y}^{*}(t):=f_{Y}\left(t, Y^{*}(t), Z^{*}(t)\right), f_{Z}^{*}(t):=f_{Z}\left(t, Y^{*}(t), Z^{*}(t)\right) .
\end{aligned}
$$

Remark 31 . Note that we do not need the concavity property of $u$ in the above theorem.

\section{Competing Interests}

The authors declare that there is no conflict of interests regarding the publication of this paper.

\section{Acknowledgments}

This work was supported by National Natural Science Foundation of China (no. 11571203) to Shaolin Ji. This work was supported by National Natural Science Foundation of China (no. 11401091) to Xiaomin Shi.

\section{References}

[1] J. Cvitanic and I. Karatzas, "Convex duality in constrained portfolio optimization," The Annals of Applied Probability, vol. 2, no. 4, pp. 767-818, 1992.

[2] I. Karatzas, J. P. Lehoczky, and S. E. Shreve, "Optimal portfolio and consumption decisions for a 'small investor' on a finite horizon," SIAM Journal on Control and Optimization, vol. 25, no. 6, pp. 1557-1586, 1987.

[3] M. Quenez, "Optimal portfolio in a multiple-priors model, Seminar on Stochastic Analysis," in Random Fields and Applications IV, pp. 291-321, Birkhäuser, Basel, Switzerland, 2004.

[4] A. Schied, "Optimal investments for robust utility functionals in complete market models," Mathematics of Operations Research, vol. 30, no. 3, pp. 750-764, 2005.

[5] N. El Karoui, S. Peng, and M. C. Quenez, "A dynamic maximum principle for the optimization of recursive utilities under constraints," The Annals of Applied Probability, vol. 11, no. 3, pp. 664-693, 2001.

[6] L. G. Epstein and S. Ji, "Ambiguous volatility and asset pricing in continuous time," The Review of Financial Studies, vol. 26, no. 7, pp. 1740-1786, 2013.

[7] L. G. Epstein and S. Ji, "Ambiguous volatility, possibility and utility in continuous time," Journal of Mathematical Economics, vol. 50, pp. 269-282, 2014.

[8] M. Hu, S. Ji, S. Peng, and Y. Song, "Backward stochastic differential equations driven by G-Brownian motion," Stochastic Processes and their Applications, vol. 124, no. 1, pp. 759-784, 2014.
[9] M. Hu and S. Ji, "Stochastic maximum principle for stochastic recursive optimal control problem under volatility ambiguity," SIAM Journal on Control and Optimization, vol. 54, no. 2, pp. 918-945, 2016.

[10] M. Hu and S. Ji, "Dynamic programming principle for stochastic recursive optimal control problem driven by a G-Brownian motion, Stochastic Processes and their Applications," https:// arxiv.org/abs/1410.3538.

[11] P. Lakner, "Optimal trading strategy for an investor: the case of partial information," Stochastic Processes and Their Applications, vol. 76, no. 1, pp. 77-97, 1998.

[12] H. Pham, "Portfolio optimization under partial observation: theoretical and numerical aspects," in Handbook of Nonlinear Filtering, Oxford University Press, 2011.

[13] J. Cvitanić, A. Lazrak, M. C. Quenez, and F. Zapatero, "Incomplete information with recursive preferences," International Journal of Theoretical and Applied Finance, vol. 4, no. 2, pp. $245-$ 261, 2001.

[14] J. Miao, "Ambiguity, risk and portfolio choice under incomplete information," Annals of Economics and Finance, vol. 10, no. 2, pp. 257-279, 2009.

[15] S. Ji and X. Y. Zhou, "A maximum principle for stochastic optimal control with terminal state constraints, and its applications," Communications in Information and Systems, vol. 6, no. 4, pp. 321-338, 2006.

[16] J. Cvitanić and I. Karatzas, "Generalized Neyman-Pearson lemma via convex duality, Bernoulli, vol. 7, no. 1, pp. 79-97, 2001.

[17] S. Ji and X. Y. Zhou, "A generalized Neyman-Pearson lemma for g-probabilities," Probability Theory and Related Fields, vol. 148, no. 3-4, pp. 645-669, 2010.

[18] S. Ji and S. Peng, "Terminal perturbation method for the backward approach to continuous time mean-variance portfolio selection," Stochastic Processes and Their Applications, vol. 118, no. 6, pp. 952-967, 2008.

[19] P. Protter, Stochastic Integration and Differential Equations, Springer, New York, NY, USA, 1990.

[20] G. Kallianpur, Stochastic Filtering Theory, Springer, New York, NY, USA, 1980.

[21] E. Pardoux and S. G. Peng, "Adapted solution of a backward stochastic differential equation," Systems and Control Letters, vol. 14, no. 1, pp. 55-61, 1990.

[22] N. El Karoui, S. Peng, and M. C. Quenez, "Backward stochastic differential equations in finance," Mathematical Finance, vol. 7, no. 1, pp. 1-71, 1997.

[23] D. Cuoco and J. Cvitanić, "Optimal consumption choices for a 'large' investor," Journal of Economic Dynamics and Control, vol. 22, no. 3, pp. 401-436, 1998.

[24] S. Peng, "Backward stochastic differential equations and applications to optimal control," Applied Mathematics and Optimization, vol. 27, no. 2, pp. 125-144, 1993.

[25] Z. Chen and L. Epstein, "Ambiguity, risk, and asset returns in continuous time," Econometrica, vol. 70, no. 4, pp. 1403-1443, 2002. 


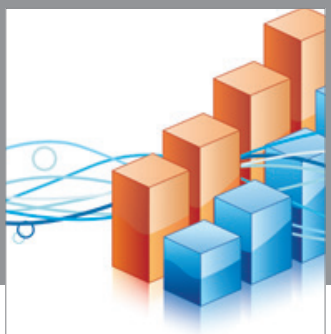

Advances in

Operations Research

vatem alat4

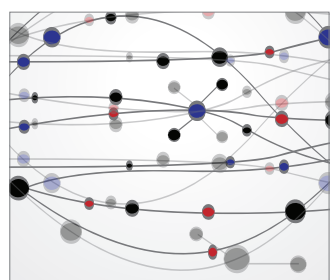

\section{The Scientific} World Journal
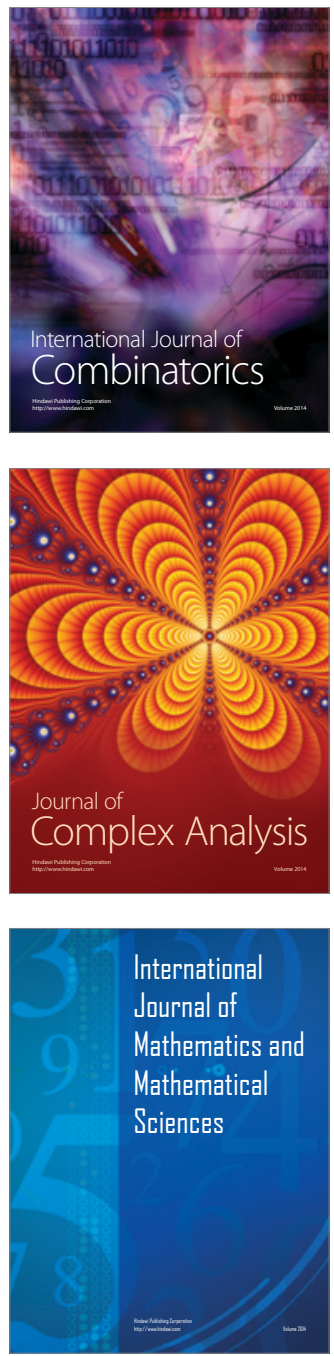
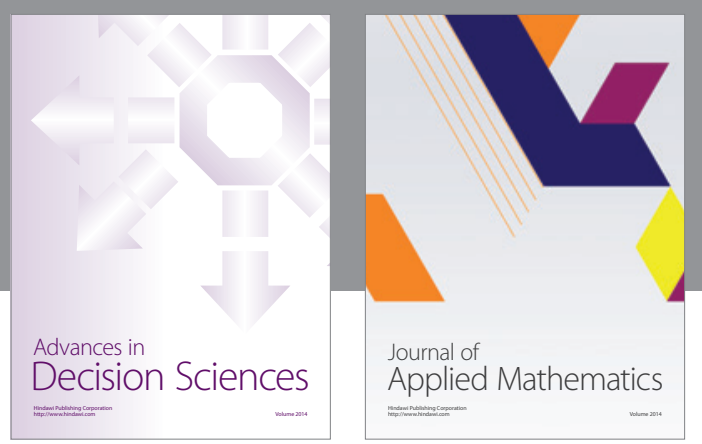

Algebra

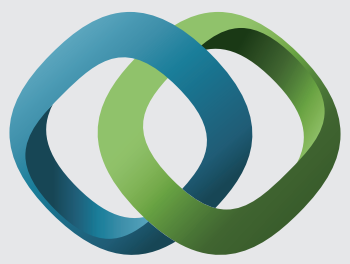

\section{Hindawi}

Submit your manuscripts at

http://www.hindawi.com
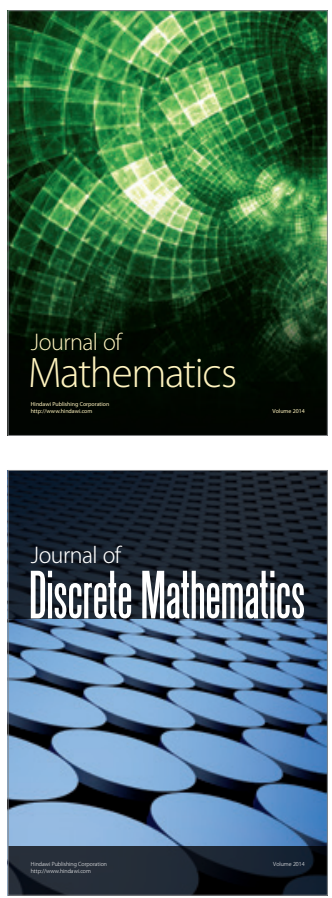

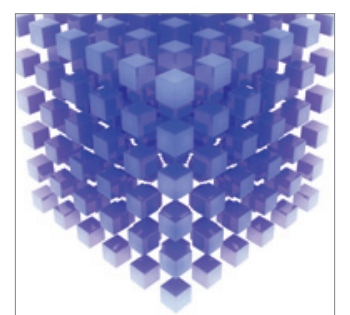

Mathematical Problems in Engineering
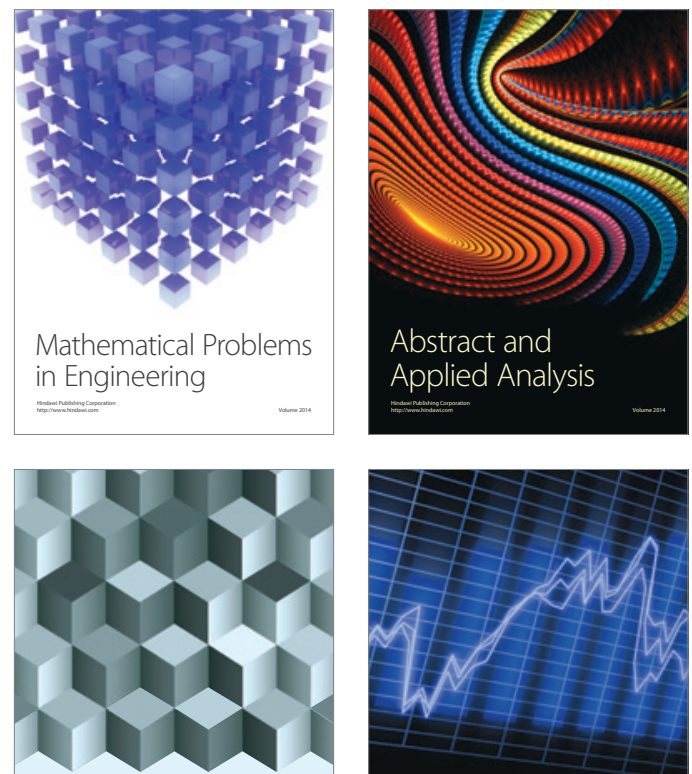

Journal of

Function Spaces

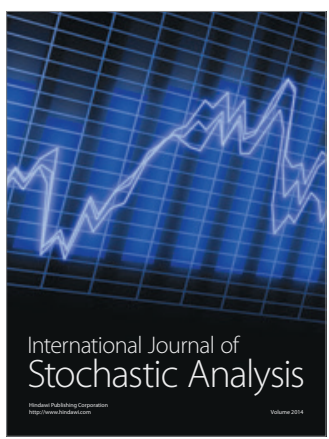

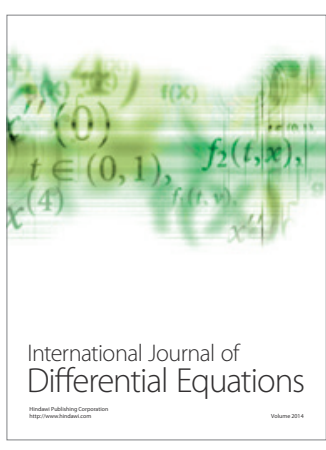
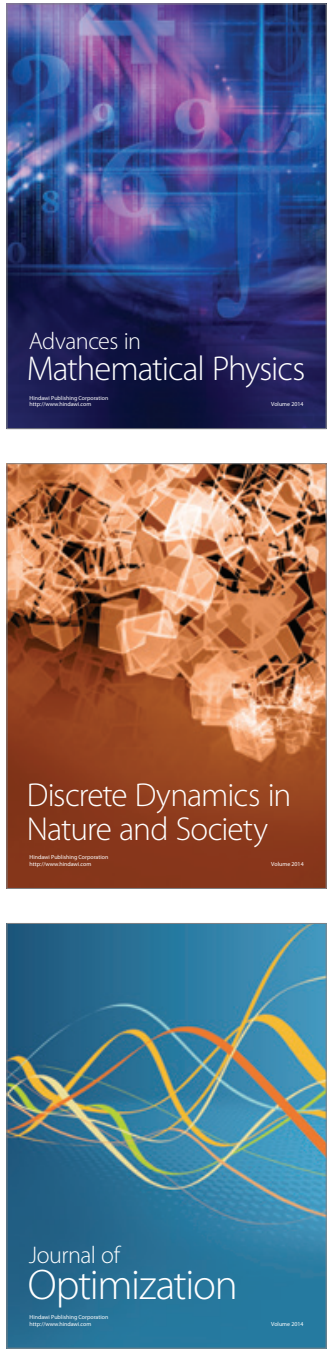